\title{
Efeitos da ciclosporina a sobre a função renal de cães da raça Golden Retriever normais ou afetados pela distrofia muscular
}

Adriana Caroprezo MORINI ${ }^{1}$ Maria Angélica MIGLINO ${ }^{1}$

Marileda Bonafim

CARVALHO ${ }^{2}$

Daniele dos Santos

MARTINS ${ }^{1}$

Carlos Eduardo AMBRÓSIO ${ }^{1}$

Carla Fabiana

CASAGRANDE $^{1}$

Alida Abatemarco CATELLI ${ }^{1}$

Ana Paula SOUZA ${ }^{2}$

Martha Maria Circhia Pinto

LUPPI $^{1}$

Thaís Peixoto GAIAD ${ }^{1}$

Mayana ZATZ $^{3}$

\section{Correspondência para:}

Adriana C. Morini - Av Prof Orlando Marques de Paiva, 87, Cidade Universitária, São Paulo-SP, 05507-000; drimorini@usp.br

Recebido para publicação: 21/11/2005 Aprovado para publicação: 07/03/2008

\author{
1 - Departamento de Cirurgia da Faculdade de Medicina Veterinária e Zootecnia \\ da Universidade de São Paulo, São Paulo-SP \\ 2 - Faculdade de Ciências Agrárias e Veterinárias da Universidade Estadual de São \\ Paulo, Jaboticabal-SP \\ 3 - Centro de Estudos do Genoma Humano do Instituto de Biologia da \\ Universidade de São Paulo, São Paulo-SP
}

\section{Resumo}

Palavras-chave: Imunossupressão.

A distrofia muscular dos cães Golden Retriever (GRMD), uma Nefrotoxicidade. miopatia degenerativa causada pela ausência da distrofina, é geneticamente homóloga à distrofia muscular de Duchenne que acomete humanos, portanto, estes cães são considerados modelos experimentais para estudos em terapia celular. Seu sucesso depende da imunossupressão adequada. A ciclosporina A é indicada para tal e a monitorização de suas concentrações sangüíneas e efeitos adversos são essenciais para viabilizar a terapia. Foram estudados cães GRMD e normais da mesma raça, submetidos à terapia com CsA, associada, nos GRMD, ao transplante de células tronco. Foram avaliados os possíveis efeitos do fármaco sobre a função renal, sendo consideradas as manifestações clínicas, urinálise, testes de função glomerular, e concentrações séricas de uréia, creatinina, sódio e potássio. Como resultado houve aumento discreto na uréia sérica de ambos os grupos; cilindrúria e proteinúria e aumento da densidade urinária no grupo dos GRMD. Concluímos que a CsA pode causar lesão aguda de túbulos renais, principalmente em GRMDs; os mesmos também reagem de modo distinto com relação à homeostase de íons e função renal. Entretanto, diagnóstico precoce e tratamento podem prevenir o agravamento das lesões e desenvolvimento de insuficiência renal.

\section{Introdução}

A distrofia muscular de Duchenne (DMD) é a mais comum desordem neuromuscular recessiva em humanos associada ao cromossomo "X", causada pela ausência da proteína distrofina que leva a uma degeneração progressiva do músculo esquelético e cardíaco e afeta apenas crianças do sexo masculino. As formas de distrofias musculares associadas ao "X", onde há ausência de distrofina, foram descritas em camundongos $m d x$, cães da raça Golden retriever e gatos. No caso do cão, a maioria dos sinais clínicos é semelhante aos sinais da DMD. Os cães afetados apresentam fraqueza progressiva e desenvolvimento de contraturas debilitantes. ${ }^{1,2,3,4,5,6,7}$

Terapias celulares envolvendo transplantes trazem esperanças para o tratamento da DMD. No entanto, enquanto a aplicação das mesmas não se torna viável, tratamentos com fármacos tornam-se cada vez mais testados. ${ }^{8}$ Atualmente as técnicas de transplantes heterólogos são amplamente difundidas sendo importante o controle da resposta imune diante da implantação do enxerto para se evitar a rejeição.'

A ciclosporina é uma gordura solúvel derivada dos fungos Tolypocladium inflatum gams $^{10,11} \mathrm{e}$, Beauveria nivea, utilizada como imunossupressor em transplante de órgãos 
para prevenir rejeições ao enxerto e como tratamento de doenças autoimunes, e também utilizada no campo da terapia gênica $^{12,13}$. Foi introduzida em 1978, para o tratamento dos transplantes alogênicos. ${ }^{11,14}$

Em se tratando de administração oral da CsA sua absorção é incompleta e depende de cada paciente individualmente. A CsA é metabolizada no fígado,em pequenas quantidades no trato gastrintestinal e nos rins. Sua eliminação primariamente é biliar e apenas $6 \%$ da dose inicial é excretada na urina sob forma de metabólitos. Sua meia vida é de 8,4 horas (oscilando entre 5 a 18 horas), sendo o pico de concentração da mesma no sangue entre 1,5 e 2 horas após administração. Apenas $0,1 \%$ da CsA é excretada pura na urina e nem mesmo a diálise pode facilitar sua excreção. Uma overdose de CsA provoca êmese forçada duas horas após sua ingestão, podendo ocorrer hepatotoxicidade e nefrotoxicidade transitórias. Dosagens muito altas provocaram morte na maioria dos ratos, coelhos e camundongos testados. ${ }^{13}$

Por se tratar de um fármaco comprovadamente hepatotóxico e nefrotóxico, o uso de CsA requer monitoração constante da creatinina sérica. Os riscos de toxicidade aumentam conforme aumenta a sua concentração sanguínea e o período de tratamento. Biópsias renais após tratamento com CsA revelaram alterações tais como vacuolização tubular, microcalcificação tubular, congestão peritubular capilar, arteriolopatias e locais com fibrose intersticial e atrofia tubular. ${ }^{13}$

Ao testar o uso da CsA em gatos, Latimer et al. ${ }^{15}$ avaliaram as funções renal e hepática, e observaram discreto, porém significante, aumento da uréia sérica, nenhuma alteração significativa na densidade urinária e redução considerável dos valores da enzima ALT.

Pesquisas também associaram CsA a outro imunossupressor ${ }^{16}$, o micofenato mofetil (MMF) e concluíram que doses baixas dos fármacos induzem tolerância imune, o que aumenta a incidência de episódios de rejeição.
Cong et al. ${ }^{17}$ concluíram em seus estudos que a associação de CsA com metotrexano resulta em uma melhora na prevenção da doença do enxerto contra hospedeiro (GVHD - graft versus host disease) em casos de transplante de células tronco hematopoiéticas de antígeno leucocitário não idêntico em modelo canino. Nesse estudo, os mesmos ainda afirmam que os fármacos utilizados, ciclosporina e metotrexano, são hematotóxicos, gastrotóxicos, nefrotóxicos, neurotóxicos e hepatotóxicos.

Em relação aos efeitos adversos, pacientes humanos apresentam misturas de grupos de sinais clínicos decorrentes de toxicidade. Em especial são citados os sinais relacionados com lesões renais, hipertensão arterial, neurotoxicidade, hepatotoxicidade e hiperplasia gengival. ${ }^{18}$

\section{Material e Método}

O projeto proposto foi conduzido após aprovação da Comissão de Bioética da Faculdade de Medicina Veterinária e Zootecnia da Universidade de São Paulo (FMVZ-USP). Foram utilizados quatro cães da raça Golden Retriever normais (GRN) e quatro afetados pela distrofia muscular (GRMD), pertencentes ao grupo de animais do canil GRMD Brasil localizado no Departamento de Cirurgia da Faculdade de Medicina Veterinária e Zootecnia da Universidade de São Paulo.

Foram considerados animais afetados pela distrofia muscular, os cães Golden Retriever do sexo masculino submetidos ao exame de genotipagem, através da extração de DNA genômico do sangue de cordão umbilical, realizado pelo Centro de Estudos do Genoma Humano da Universidade de São Paulo. O exame compreende a identificação da mutação de ponto, do sítio 3' de splice, do intron 6 do gene da distrofina. Essa mutação leva à perda do exon 7 durante o processamento do mRNA, gerando um stop códon do exon 8 e um peptídeo com 5\% do tamanho normal da proteína distrofina. Os cães considerados normais são submetidos ao mesmo processo de exame 
de genotipagem, porém sem constar a mutação a que se refere à doença.

Os animais GRMD receberam ciclosporina A e foram submetidos ao transplante de células tronco de cordão umbilical. Os animais GRN receberam somente tratamento com ciclosporina A. O imunossupressor empregado teve sua dose variando entre $1 \mathrm{mg} / \mathrm{kg}$ e $10 \mathrm{mg} / \mathrm{kg}$, a cada doze horas, por um período de 32 dias para os cães normais e de até 67 dias para os transplantados. As doses foram ajustadas, caso a caso, ao longo do tratamento na tentativa de manter concentrações séricas variando entre 250 a $500 \mathrm{ng} / \mathrm{L}$. As concentrações foram adquiridas por meio de colheita de amostras de sangue, duas vezes por semana, minutos antes da administração de CsA pela manhã, ou seja, doze horas após a administração da última dose. As mesmas foram encaminhadas ao laboratório de Análises Clínicas do Hospital Israelita Albert Einstein. O transplante celular (somente para os G-GRMD) foi realizado pela equipe do IB-USP e da FMVZ-USP, de acordo com protocolo estabelecido em projeto próprio.

No dia um do experimento, antes de qualquer administração de CsA ou do transplante, foram realizadas todas as avaliações propostas e seus resultados considerados como valores basais (controle). Ao longo do período de tratamento, iniciado no dia dois do experimento (duração de 30 ou de até 67 dias) foram avaliados os parâmetros relativos à função renal que consistiram em: análise físico-química da urina, concentração sérica de uréia, creatinina, sódio e potássio e razão proteína: creatinina da urina, todos esses exames foram realizados duas vezes por semana, sendo o exame físico e urinálise feitos diariamente; o momento da colheita das amostras foi determinado sempre doze horas após a administração do fármaco.

As amostras de urina foram colhidas por meio de micção espontânea (jato médio) e os exames foram realizados imediatamente após a coleta, nas próprias dependências do Canil GRMD - Brasil. A densidade urinária foi determinada por meio de refratometria
(Refratômetro UGI (1000-1050) Atago). O $\mathrm{pH}$ urinário foi determinado através de pHmetro (Phmetro de bolso Q-400 BD QUIMIS). Os demais parâmetros bioquímicos da urinálise de rotina foram mensurados por meio de tiras reagentes (COMBUR $\stackrel{10}{ }$ TEST - Boehringer Mannheim Argentina S.A., Buenos Aires). A sedimentoscopia foi feita em microscópio (Nikon eclipse E-200) com aumento de 250 a 400x, após centrifugação (Centrífuga Celm LS3 plus) da urina.

As análises bioquímicas séricas e urinárias foram feitas em amostras do soro obtidas após sinérese de sangue coletado por punção das veias jugular ou cefálica, no Laboratório de Patologia Clínica da UNESP - Jaboticabal.

Utilizando o sistema Labtest (LABTEST Diagnóstica S.A. - Lagoa Santa, $\mathrm{MG}$ ) para diagnóstico, alíquotas das amostras de soro foram processadas para determinação de creatinina [método de Jaffé (Método do picrato alcalino ) modificado]; uréia (UV-cinética - pelo método do diacetil modificado); sódio e potássio (eletrodo íonseletivo). Nas amostras de urina foram determinadas as concentrações de creatinina (método cinético - Jaffé modificado); proteína (método vermelho de pirogalol sensiprot); e uréia (método da uréase). As leituras foram realizadas em dosador semiautomático para provas bioquímicas, considerando-se a faixa visível para as respectivas provas.

O clearance de creatinina endógena, empregado para estimar a taxa de filtração glomerular, foi feito por meio da técnica de dois períodos subseqüentes de 20 minutos, de acordo com o método descrito por Souza et al. ${ }^{19}$, nas próprias dependências do Canil GRMD - Brasil. A avaliação da razão proteína/creatinina da urina foi avaliada ${ }^{20}$ no Laboratório de Patologia Clínica da UNESP de Jaboticabal. Os resultados obtidos foram analisados pelo método da análise de variância e por comparação de médias. $\mathrm{O}$ sistema utilizado foi o Statistical Analysis System versão 9.0.

Todos os animais receberam 
acompanhamento e os cuidados médicos que se fizeram necessários em função do transplante e dos efeitos colaterais do imunossupressor. Para tanto os animais foram assistidos ininterruptamente (24 horas por dia) por um ou mais membros da equipe em regime de plantões de seis a oito horas. Os cuidados regulares incluíram procedimentos de higiene, alimentação, administração da CsA e avaliação clínica.

Foram avaliados diariamente o peso corporal, ingestão de água e alimento, freqüências cardíaca e respiratória, temperatura interna, hidratação, estado geral, defecação e qualidade das fezes, micção e qualidade da urina. Exame físico completo foi executado caso fossem notadas quaisquer alterações. Quando necessário os animais receberam fluidoterapia, além de tratamento sintomático e de suporte; como por exemplo, em quadros de desidratação; êmese e diarréia, atribuídos ao fármaco; verminose, adquirida devido à baixa do sistema imune; anemias, cilindrúria, gengivite, seborréia, intoxicação pela CsA, entre outras.

\section{Resultados}

Ao longo do tratamento foi observado que os Golden Retriever estudados apresentaram diminuição do apetite, em grau leve.

Os valores séricos de creatinina e a razão proteína/creatinina da urina não variaram entre os grupos nem em função do tempo de tratamento. No que diz respeito à uréia sérica, embora tenham sido detectadas diferenças (Pd"0,01) em relação ao tempo, os valores obtidos estavam dentro do padrão de normalidade não havendo significado clínico para o achado. Os resultados são visualizados na tabela 1.

A taxa de filtração glomerular estimada por meio do clearance de creatinina não variou de forma significativa em função dos grupos ou do tempo e, todos os resultados obtidos mantiveram-se dentro dos valores de referência para a espécie. O mesmo foi observado para os valores de concentração sérica de sódio. Os valores de potássio sérico diferiram (Pd"0,05) nos tempos 11, 15 e 18 entre os grupos e entre os tempos 15 e 29 no grupo dos GRMD (Tabela 2).

Os resultados das urinálises, interpretados individualmente, revelaram alterações indicativas de lesões renais manifestadas após o início da terapia imunossupressora. Os resultados referentes aos parâmetros densidade, $\mathrm{pH}$, e presença de proteínas e cilindros na urina dos cães normais e afetados estão apresentados na tabela 3 .

\begin{tabular}{|c|c|c|c|c|c|c|}
\hline \multirow[t]{2}{*}{ Dias } & \multicolumn{2}{|c|}{ Creatinina sérica } & \multicolumn{2}{|c|}{ Uréia sérica } & \multicolumn{2}{|c|}{ Razão proteína/creatinina (UP:C) } \\
\hline & GRN & GRMD & GRN & GRMD & GRN & GRMD \\
\hline 1 & $0,72 \pm 0,12$ & $0,76 \pm 0,18$ & $15,66 \pm 3,78$ & $27,66 \pm 3,78$ & $0,33 \pm 0,10$ & $0,58 \pm 0,47$ \\
\hline 4 & $0,78 \pm 0,14$ & $0,71 \pm 0,17$ & $26,75 \pm 4,85$ & $23,25 \pm 2,62$ & $0,32 \pm 0,23$ & $0,50 \pm 0,50$ \\
\hline 8 & $0,73 \pm 0,11$ & $0,56 \pm 0,09$ & $34,25 \pm 14,52$ & $38,00 \pm 12,28$ & $0,33 \pm 0,20$ & $0,47 \pm 0,45$ \\
\hline 11 & $0,71 \pm 0,13$ & $0,68 \pm 0,13$ & $30,25 \pm 11,26$ & $32,50 \pm 3,32$ & $0,20 \pm 0,11$ & $0,47 \pm 0,36$ \\
\hline 15 & $0,76 \pm 0,13$ & $0,71 \pm 0,15$ & $34,00 \pm 16,30$ & $38,50 \pm 13,69$ & $0,19 \pm 0,10$ & $0,62 \pm 0,68$ \\
\hline 18 & $0,78 \pm 0,20$ & $0,71 \pm 0,18$ & $35,00 \pm 11,22$ & $33,75 \pm 8,38$ & $0,30 \pm 0,06$ & $0,88 \pm 0,68$ \\
\hline 22 & $0,89 \pm 0,08$ & $0,68 \pm 0,11$ & $32,33 \pm 16,28$ & $30,25 \pm 4,92$ & $0,18 \pm 0,08$ & $0,97 \pm 1,02$ \\
\hline 25 & $0,70 \pm 0,15$ & $0,73 \pm 0,22$ & $34,75 \pm 16,87$ & $23,25 \pm 1,89$ & $0,49 \pm 0,35$ & $0,79 \pm 0,43$ \\
\hline \multirow[t]{2}{*}{29} & $0,78 \pm 0,16$ & $0,61 \pm 0,19$ & $30,00 \pm 12,00$ & $35,00 \pm 15,47$ & $0,28 \pm 0,13$ & $0,61 \pm 0,42$ \\
\hline & \multicolumn{2}{|c|}{ C.V. } & F grupos & \multicolumn{2}{|c|}{ F tempo } & upo $x$ tempo \\
\hline Creatinina & \multicolumn{2}{|c|}{$14,71 \%$} & $0,49^{\mathrm{NS}}$ & \multicolumn{2}{|c|}{0,59 NS } & $1,11^{\text {NS }}$ \\
\hline Uréia & \multicolumn{2}{|c|}{$22,02 \%$} & $0,02^{\mathrm{NS}}$ & \multicolumn{2}{|c|}{$2,83^{*}$} & $1,95^{\mathrm{NS}}$ \\
\hline UP:C & \multicolumn{2}{|c|}{$48,45 \%$} & $1,57^{\mathrm{NS}}$ & \multicolumn{2}{|c|}{$1.12^{\mathrm{NS}}$} & $1,18^{\mathrm{NS}}$ \\
\hline
\end{tabular}


Tabela 2 - Médias, desvios- padrão e resultados da análise de variância dos valores de clearance de creatinina $(\mathrm{mL} / \mathrm{min} / \mathrm{kg})$; uréia sérica $(\mathrm{mg} / \mathrm{dL})$ e razão proteína/creatinina da urina de cães Golden Retriever submetidos à imunossupressão com ciclosporina $\mathrm{A}$, normais $(\mathrm{GRN}, \mathrm{n}=4)$ e afetados pela distrofia muscular progressiva (GRMD, $\mathrm{n}=4)$ - São Paulo - 2005

\begin{tabular}{|c|c|c|c|c|c|c|}
\hline & \multicolumn{2}{|c|}{$\begin{array}{l}\text { Clearance de creatinina } \\
(\mathrm{mL} / \mathrm{min} / \mathrm{kg})\end{array}$} & \multicolumn{2}{|c|}{ sódio sérico $(\mathrm{mg} / \mathrm{dL})$} & \multicolumn{2}{|c|}{ potássio sérico $(\mathrm{mg} / \mathrm{dL})$} \\
\hline & GRN & GRMD & GRN & GRMD & GRN & GRMD \\
\hline 1 & $1,87 \pm 1,03$ & $2,95 \pm 1,35$ & $153,00 \pm 10,81$ & $136,00 \pm 4,58$ & $5,26 \pm 0,20$ & $4,50 \pm 0,60$ \\
\hline 4 & & $2,70 \pm 1,17$ & $150,25 \pm 14,22$ & $139,25 \pm 1,89$ & $5,05 \pm 0,30$ & $4,42 \pm 0,71$ \\
\hline 8 & & & & $136,00 \pm 6,24$ & & $5,03 \pm 0,25$ \\
\hline 11 & $2,63 \pm 0,96$ & & $150,00 \pm 7,00$ & $144,33 \pm 8,50$ & $5,43 \pm 0,20 \mathrm{~A}$ & $4,06 \pm 0,75^{\text {B }}$ \\
\hline 15 & & $2,66 \pm 1,95$ & $145,00 \pm 2,82$ & $142,25 \pm 1,25$ & $5,25 \pm 0,21 \mathrm{~A}$ & $4,15 \pm 0,45^{\mathrm{Ba}}$ \\
\hline 18 & $2,36 \pm 0,58$ & & $151,00 \pm 5,56$ & $147,00 \pm 3,60$ & $5,23 \pm 0,05 \mathrm{~A}$ & $4,43 \pm 0,73^{\text {B }}$ \\
\hline 22 & & & $144,66 \pm 6,11$ & $152,00 \pm 11,28$ & $5,23 \pm 0,15$ & $5,05 \pm 0,81$ \\
\hline 25 & $2,67 \pm 0,56$ & $2,91 \pm 0,75$ & $150,00 \pm 5,71$ & $148,00 \pm 11,35$ & $5,02 \pm 0,27$ & $4,90 \pm 0,26$ \\
\hline \multirow[t]{2}{*}{29} & $2,94 \pm 0,38$ & $2,89 \pm 0,36$ & $148,00 \pm 10,36$ & $155,75 \pm 17,96$ & $4,92 \pm 0,29$ & $5,37 \pm 0,41^{\mathrm{b}}$ \\
\hline & & C.V. & \multicolumn{2}{|c|}{ F grupos $\quad$ F } & & upo $x$ tempo \\
\hline \multicolumn{2}{|c|}{ clearance } & $25,36 \%$ & \multicolumn{2}{|c|}{$0,83^{\mathrm{NS}}$} & & $0,98^{\mathrm{NS}}$ \\
\hline \multicolumn{2}{|c|}{ Sódio } & $5,55 \%$ & \multicolumn{2}{|c|}{$0,17^{\mathrm{NS}}$} & & $1,57^{\mathrm{NS}}$ \\
\hline \multicolumn{2}{|c|}{ Potássio } & $8,98 \%$ & \multicolumn{2}{|c|}{$4,07 \mathrm{NS}$} & & $2,34^{*}$ \\
\hline
\end{tabular}

* $\mathrm{P} \leq 0,05 . \mathrm{NS}$ - não significativo. Médias seguidas de letras maiúsculas distintas em linha, e minúsculas em coluna diferem entre si pelo teste de Tukey-Kramer.

Obs: As lacunas em branco compreendem falha em algum dos pontos do processo de análise, que implicou na ausência de resultado final

Tabela 3 - Médias e desvios-padrão das densidades, pH, proteína e cilindros na urina dos cães normais e afetados pela distrofia muscular decorrentes de todo o período de experimento (60 dias para GRMD e 30 dias para GRN) submetidos a imunossupressão com ciclosporina A - São Paulo - 2005

\begin{tabular}{ccccccccc}
\hline & & Densidade & $\mathrm{pH}$ & $\begin{array}{c}\text { Proteína } \\
(\mathrm{mg})\end{array}$ & $\begin{array}{c}\text { Cilindro } \\
\text { hialino* }\end{array}$ & $\begin{array}{c}\text { Cilindro } \\
\text { celular* }\end{array}$ & $\begin{array}{c}\text { Cilindro } \\
\text { granuloso* }\end{array}$ & $\begin{array}{c}\text { Cilindro } \\
\text { céreo* }\end{array}$ \\
\cline { 3 - 8 } $\begin{array}{c}\text { desvio } \\
\text { padrão }\end{array}$ & GRN 1 & $1,021 \pm 1,010$ & $6,8 \pm 1,3$ & $6,56 \pm 12,60$ & $0,03 \pm 0,18$ & $0,0 \pm 0,0$ & $0,03 \pm 0,18$ & $0,0 \pm 0,0$ \\
& GRN 2 & & $6 \pm 1$ & $0,0 \pm 0,0$ & $0,16 \pm 0,40$ & $0,0 \pm 0,0$ & $0,16 \pm 0,40$ & $0,0 \pm 0,0$ \\
& GRN 3 & & $5,2 \pm 0,3$ & $0,0 \pm 0,0$ & $0,0 \pm 0,0$ & $0,0 \pm 0,0$ & $0,33 \pm 0,81$ & $0,0 \pm 0,0$ \\
& GRN 4 & & $6 \pm 1,2$ & $0,0 \pm 0,0$ & $0,0 \pm 0,0$ & $0,0 \pm 0,0$ & $0,0 \pm 0,0$ & $0,0 \pm 0,0$ \\
& GRMD 5 & $1,021 \pm 0,015$ & $6,4 \pm 1$ & $19,78 \pm 28,79$ & $0,17 \pm 0,52$ & $0,06 \pm 0,25$ & $0,76 \pm 1,03$ & $0,0 \pm 0,0$ \\
& GRMD 6 & $1,054 \pm 0,020$ & $5,9 \pm 0,8$ & $22,94 \pm 28,34$ & $0,46 \pm 0,87$ & $0,03 \pm 0,17$ & $0,21 \pm 0,49$ & $0,03 \pm 0,17$ \\
& GRMD 7 & $1,016 \pm 0,009$ & $6,9 \pm 1,2$ & $4,85 \pm 11,22$ & $0,11 \pm 0,39$ & $0,0 \pm 0,0$ & $0,0 \pm 0,0$ & $0,0 \pm 0,0$
\end{tabular}

* escore de cilindros: $0=$ negativo; $1=$ raros; $2=1+; 3=2+; 4=3+$;

Obs: As lacunas em branco compreendem falha em algum dos pontos do processo de análise, que implicou na ausência de resultado final

\section{Discussão}

Os animais apresentaram diminuição do apetite, em grau leve, ao longo do tratamento, onde um único animal apresentou episódios de vômito e diarréia durante um período de 12 horas, o qual correspondeu a um dos picos de concentração sérica de CsA observados. Em gatos, as doses baixas de CsA podem induzir imunotolerância como descrito por George et al. ${ }^{16}$, Por outro lado, doses mais altas são acompanhadas de reações adversas tais como anorexia, diarréia e vômitos.

De acordo com dados descritos em pesquisas anteriores, baseadas em estudos em seres humanos ${ }^{11,18,21,22,23,24}$, cães ${ }^{17}$ e gatos ${ }^{15}$, a CsA é nefrotóxica e hepatotóxica. No presente estudo, foram detectados sinais de lesão de túbulos renais, caracterizados por aumento da perda de proteína na urina e cilindrúria, principalmente nos cães GRMD. Entretanto, como evidenciado pelos resultados das provas de função, os animais 
não chegaram a desenvolver insuficiência renal. Provavelmente, as medidas terapêuticas instituídas precocemente foram efetivas, tais como fluidoterapia com diferentes soluções, aplicação de diuréticos, instituição de alimentação mais úmida (acréscimo de água), realizadas logo após o resultado da urinálise diária, quando observadas desidratação, cilindrúria, oligúria entre outros.

Considerando as características clínicas peculiares aos animais com distrofia muscular, pode-se inferir que a doença seja um fator predisponente para o desencadeamento da agressão renal determinada pela CsA. Contudo, não foram encontrados outros relatos sobre avaliação da função renal de cães Golden Retriever com Distrofia Muscular.

Com relação ao íon sódio não foram identificadas alterações decorrentes da administração de CsA e os valores observados encontravam-se dentro dos padrões normais ${ }^{25}$ para cães jovens. Todavia, os valores de potássio sérico, embora estivessem dentro do padrão de normalidade para a espécie tenderam a ser menores nos GRMDs, não havendo explicação, até o presente estudo, para tal ocorrência.

\section{Conclusões}

$\mathrm{Nas}$ condições do presente experimento, a terapia com CsA pode causar lesão aguda de túbulos renais, dos Golden Retriever afetados por distrofia muscular. Entretanto, diagnóstico precoce e tratamento adequado podem preveniram o agravamento das lesões e desenvolvimento de insuficiência renal.

Os cães da raça Golden Retriever afetados pela distrofia muscular progressiva, no que se refere aos efeitos colaterais, à homeostase de íons e à função renal, reagiram de modo distinto dos indivíduos normais.

\section{Agradecimentos}

À Fundação de Amparo a Pesquisa de São Paulo pelo apoio financeiro.

\section{Effect of cyclosporin A on renal functions of normal Golden Retriever dogs or Golden Retriever with muscular dystrophy}

\begin{abstract}
The muscular dystrophy of Golden Retriever is a degenerative miopaty caused by the absence of dystrophy and it is genetically homologue of the Duchenne muscular dystrophy in humans, so, these dogs are considerably experimental models for studies on cellular therapy. Their successful depends of the adequate immunosuppression. Cyclosporin $\mathrm{A}$ is indicated for that and the monitoring of blood concentration and adverse effects are essential to viabilise the therapy. It was studied GRMD dogs, and normal dogs from the same breed, submitted for therapy with CsA, associated, on GRMD, of cell transplantation. It was evaluated the possible effects of the drug on renal functions. It has been considerate the clinic manifestations, urinalisis, testis of glomerular function and blood concentrations of urea, cretinine, sodium and potassium. In our results we found a discrete increase of blood urea on booth groups; increased levels of urine's cylinders and protein and also increase of urinary density on GRMD group. CsA therapy could make acute lesions on renal tubules, especially on GRMD. These dogs also have different reactions than normal dogs on relation of ions homeostasis and renal function. However, earlier diagnosis and adequate treatment could prevent the development of renal diseases.
\end{abstract}

Key words: Immunosuppression. Nefrototoxicity. 


\section{Referências}

1 BERGMAN, R. L.; INZANA, K. D.; MONROE, W. E.; SHELL, L. G.; LIU, L. A.; ENGVALL, E.; SHELTON, G. D. Dystrophin-deficient muscular dystrophy in a Labrador retriever. Journal of American Hospital Association, v. 38, n. 3, p. 255-261, 2002.

2 BOGDANOVICH, S.; PERKINS, K. J.; KRAG, T. O. B.; KHURANA, T. S. Therapeutics for Duchenne muscular dystrophy: current approaches and future directions. Journal of Molecular Medicine, v. 82, n. 2, p. 102-115, 2004.

3 CHILDERS, M. K.; OKAMURA, C. S.; BOGAN, D. J.; BOGAN, J. R.; PETROSKI, G. F.; MCDONALD, K.; KORNEGAY, J. N. Eccentric contraction injury in dystrophic canine muscle. Archieve of Physical Medicine Rehabilitation, v. 83, p. 1572-1578, 2002.

4 KORNEGAY, J. N.; BOGAN, D. J.; BOGAN, J. R.; CHILDERS, M. K.; CUNDIFF, D. D.; PETROSKI, G. F.; SCHUELER, R. O. Contraction force generated by tarsal joint flexion and extension in dogs with Golden Retriever muscular dystrophy. Journal of the Neurological Sciences, v. 166, p. 155-121, 1999.

5 LIU, J. M. K.; OKAMURA, C. S.; BOGAN, D. J.; BOGAN, J. R.; CHILDERS, M. K.; KORNEGAY, J. N. Effects of prednisone in canine muscular dystrophy. Muscle \& Nerve, v. 30, p. 767-773, 2004.

6 NGUYEN, F.; GUIGAND, L.; GOUBAULTLEROUX, I.; WYERS, M.; CHEREL, Y. Microvessel density in muscles of dogs with Golden Retriever muscular dystrophy. Neuromuscular Disorders, v. 15, p. 154-163, 2005.

7 SHELTON, G. D.; LIU, L. A.; GUO, L. T.; SMITH, G. K.; CHRISTIANSEN, J. S.; THOMAS, W. B.; SMITH, M. O.; KLINE, K. L.; MARCH, P. A.; FLEGEL, T.; ENGVALL, E. Muscular dystrophy in female dogs. Journal Veterinary Internal Medicine, v. 15, n. 3, p. 240-244, 2001.

8 MARQUES, M. J.; LUZ, M. A. M.; MINATEL, E.; SANTO NETO, $\mathrm{H}$. Muscle regeneration in dystrophic $m d x$ mice is enhanced by isosorbide dinitrate. Neuroscience Letters, v. 382, p. 342-345, 2005.

9 FIRST, M. R. Immunosupressive agents and their actions. Transplantation Proceedings, v. 34, p. 13691372, 2002

10 GONÇALVES, P. F.; NOGUEIRA FILHO, G. R.; SALLUM, E. A.; SALLUM, A. W.; NOCITI JUNIOR, F. $\mathrm{H}$. Immunosuppressant therapy and bone loss in ligature-induced periodontitis - a study in rats. Pesquisa Odontológica Brasileria, v. 17, n. 1, p. 46-50, 2003.

11 LIN-WANG, H. T.; MANRIQUE, R. Aplicação da técnica de imunoensaio enzimático de multiplicação (EMIT) para dosagem de ciclosporina na amostra de sangue absorvido em papel filtro. Jornal Brasileiro de Patologia e Medicina Laboratorial, v. 38, n. 1, p. 7-12,
2002.

12 O'HARA, A. J.; COLLINS, T.; HOWELL, J. M. C. Gingival eruption cysts induced by cyclosporine administration to neonatal dogs. Journal of Clínical Periodontology, v. 29, p. 507-513, 2002.

13 NOVARTIS PHARMA. Neoral ${ }^{\circledR}$. Soft geltin capsules. Neoral - cyclosporine. Apresenta dados de literatura sobre o imunossupressor ciclosporina $\mathrm{A}$, texto técnico científico. Disponível em: <www.pharma.us.novartis.com/product/pi/pdf/ neoral.pdf >. Acesso em: 14 fev. 2004.

14 MURDAN, S.; ANDRÝSEC, T.; SON, D. Nove gels and their dispersions-oral drug delivery systems for ciclosporin. International Journal of Pharmaceutics, v. 300, p. 113-124, 2005.

15 LATIMER, K. S.; RAKICH, P. M.; PURSWELL, B. J.; KIRCHER, I. M. Effects of cyclosporin A administration in cats. Veterinary Immunology and Immunopathology, v. 11, p. 161-173, 1986.

16 GEORGES, E. G.; STORB, R.; THOMPSON, J. D.; YU, C.; GOOLEY, T.; BRUNO, B.; NASH, R. A. Adoptive immunotherapy in canine mixed chimeras after nonmyeloablative hematopoietic cell transplantation. Blood, v. 95, n. 10, p. 3262-3269, 2000.

17 CONG, Y.; LINSLEY, P.; SEIDEL, K.; SALE, G.; DEEG, J. H.; NASH, R.; STORB, R. Cytotoxic T lymphocyte antigen 4-immunoglobulin fusionprotein combined wiyh methotrexate/cyclosporine as graft-versus-hostdisease prevention in a canine dog leucocyte antigennonidentical marrow transplant model. Transplantation, v. 69, n. 3 , p. $450-454,2000$.

18 BOWERS, L. D. Therapeutic monitoring for cyclosporine: difficulties in establishing a therapeutic window. Clinical Biochemistry, v. 24, p. 81-87, 1991.

19 SOUZA, A. P. Contribuição ao estudo dos métodos para a determinação do clearance de creatinina em cães - resultados preliminares. In: CONGRESSO BRASILEIRO DE CLÍNICOS DE PEQUENOS ANIMAIS, 20. 1999, Águas de Lindóia, Anais... Águas de Lindóia: Associação nacional de Clínicos de Pequenos Animais, 1999. p. 34

20 FINCO, D. R. Evaluation of renal functions In: OSBORNE, C. A.; FINCO, D. R. (Ed.). Canine and feline nephrology and urology. Baltimore: Williams \& Wilkins, 1995. p. 216-219.

21 JUSTO, P.; LORZ, C.; SANZ, A.; EGIDO, J.; ORTIZ, A. Intracellular mechanisms of Cyclosporin A - induced tubular cell apoptosis. Journal of American Society of Nephrology, v. 14, p. 3072-3080, 2003.

22 CARAMELO, C.; ALVAREZ-ARROYO, M. V.; YAGÜE, S.; SUZUKI, Y.; CASTILLA, M. A.; VELASCO, L.; GONZALEZ-PACHECO, F. R.; TEJEDOR, A. Cyclosporin A toxicity, and more: vascular endothelial growth factor (VEGF) steps forward. Nephrology Dialysis Transplantation, v. 19, p. 285-288, 2004. 
23 PÉREZ, M.; CASTILLA, M.; TORRES, A. M.; LÁZARO, J. A.; SARMIENTO, E.; TEJEDOR, A. Inhibition of brush border dipeptidase with cilastatin reduces toxic accumulation of cyclosporin A in kidney proximal tubule epithelial cells. Nephrology Dialysis Transplantation, v. 19, p. 2445-2455, 2004.

24 HORTELANO, S.; CASTILLA, M.; TORRES, A. M.; TEJEDOR, A.; BOSCÁ, L. Potentiation by nitric oxide of Cyclosporin A and FK506-Induced apoptosis in renal proximal tubule cells. Journal of American Society of Nephrology, v. 11, p. 2315-2323, 2000.

25 MEYER, D. J.; COLES, E. H.; RICH, L. J. Veterinary laboratory medicine. Philadelphia: W. B. Saunders, 1992. p. 27-120. 